Journal of Scientific Perspectives

Volume 3, Issue 1, Year 2019, pp. 69-84

E - ISSN: 2587-3008

URL: http://ratingacademy.com.tr/ojs/index.php/jsp

DOİ: $10.26900 /$ jsp.3.008

Research Article

\title{
RARE AND ENDEMIC TAXA OF LAMIACEAE IN TURKEY AND THEIR THREAT CATEGORIES*
}

\author{
Gül KUSSAKSIZ * \\ * Assoc. Prof. Dr., Bursa Uludă̆ University, Faculty of Science and Arts Department of Biology, \\ TURKEY,E-mail: gult@uludag.edu.tr, \\ ORCID ID: https://orcid.org/0000-0001-2345-6789
}

Received: 6 January 2019; Accepted: 26 January 2019

\begin{abstract}
Lamiaceae (Labiatae) is the third largest family in Turkey. It is represented by 48 genera and 782 taxa (603 species, 179 subspecies and varieties) in the country, 346 taxa ( 271 species, 75 subspecies and varieties) of which are endemic. Endemism ratio is ca. \% 44. The largest genera in the country based on the taxon number are Stachys L.(118 taxa), Salvia L.(107 taxa), Sideritis L.(54 taxa), Phlomis L.(53 taxa), Teucrium L. (49 taxa), Thymus L.(47 taxa). Endangered genera according to IUCN criteria; Salvia L.(43 taxa), Stachys L.( 19 taxa) Thymus L. (16 taxa), Sideritis L.(14 taxa), Teucrium L. (10 taxa), Lamium L.(8 taxa), Phlomis L. and Scutellaria L. ( 7 taxa), Micromeria Bentham and Nepeta L. ( 6 taxa), Ajuga L.and Origanum L. (5 taxa), Marrubium L..( 4 taxa), Acinos Miller, Satureja L. and Calamintha Miller (2 taxa), Ballota L., Dorystaechas Boiss. \& Heldr. ex Bentham, Cyclotrichium (Boiss.) Manden. \& Scheng. and Lopanthus Adans. (1 taxon). Lamiaceae has 160 taxa endangered in Turkey of which are categories; 43 taxa critically endangered, 59 taxa endangered and 58 taxa vulnerable. Phytogeographic distribution of rare and endemic Turkish Lamiaceae taxa are 79 taxa in the Mediterranean, 63 taxa in the Irano-Turanien, 5 taxa in the Euro-Siberian phytogeographic region, and 11 taxa in unknown or multiregional phytogeographical elements.This study was carried out to draw attention to the endangered and vulnerable taxa in Lamiaceae family and to indicate which phytogeographic regions these taxa are more widely distributed.
\end{abstract}

Keywords: Lamiaceae, Threat categories, Turkey.

\section{INTRODUCTION}

Turkey is among the richest countries in the World in terms of plant diversity. With around 11.707 flowering plant species, the flora is the richest of any country in Europe, North Africa and Middle East. (Davis 1982, Davis et al. 1988, Güner et al. 2000, Güner 2012).

Wild Flowering Plants in Turkey (Güner 2012)

\footnotetext{
* The first version of this paper is presented in the" 2nd International Conference on Awareness" held in Kepez/Çanakkale on December 13-15, 2018.
} 


\begin{tabular}{|c|c|c|c|c|c|}
\hline Family & $\underline{\text { Genera }}$ & Species & $\underline{\text { Subspecies }}$ & $\underline{\text { Varietes }}$ & Total infrageneric taxa \\
\hline 167 & 1320 & 9996 & 1989 & 867 & 11707 \\
\hline
\end{tabular}

The main reasons for this wealth are as follow;

-A variety of climates

-Topographical diversity with marked changes in ecological factors over short distance

-Geological and geomorphic variation

-A range of aquatic environments such as seas,lakes and rivers

- Altitude variations from sea level to 5000 (Ekim and Güner 2000).

The situation of the country at the junction of three major phytogeographical regions:

-Mediterranean

-Irano-Turanian

-Euro-Siberian

There are the number of major mountain ranges in Anatolia which constitute effective barriers and these have further encouraged a greater diversity of species. Additionally, during many historical periods, Anatolia has served as a passege way between the continents of Europe, Asia and Africa, resulting in dispersal of a wide variety of plants and animals (Anonim 1996). All these factors combined have provided many opportunities for the plants to evolve and differentiate creating the present wealth of species now estimated to be over 11.707 taxa, about one third of which are endemic (3649 taxa) (Güner 2012). Lamiaceae is the third largest family in Turkey. The family has 48 genera and 782 taxa, 346 taxa of which are endemic ( ca. $44 \%$ ) in the country (Celep and Dirmenci 2017).

Figure 1. Phytogeographic regions of Turkey (Davis, 1965; modified byAvc1, 1996).

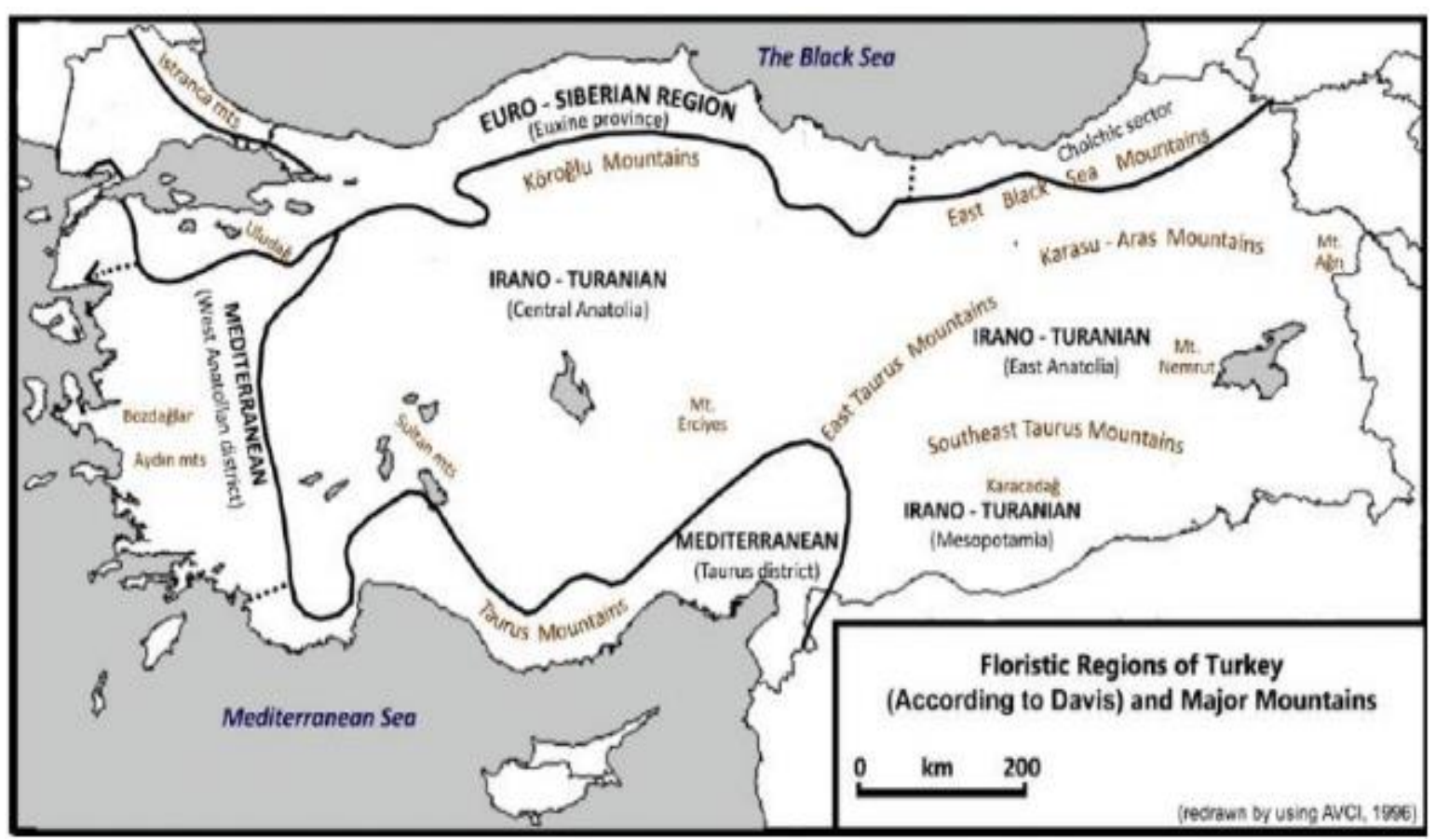


The purpose of this study are to obtain the threatened category of endemic and rare taxa of Lamiaceae family in Turkey and to indicate which phytogeographic regions these taxa are more widely distributed.

\section{MATERIAL AND METHODS}

Data were obtained by scanning Flora of Turkey, Red Data Books, The List of Turkey Plants (Vascular Plants), check-lists, publications and observations (Davis 1982, Davis et al. 1988, Güner et al. 2000, Tarımcılar 1998, Aytaç and Aksoy 2000, Ekim et al. 2000, Dönmez 2001, Dirmenci 2003, Akçiçek and Vural 2007, Alan 2009, Ekim 2009, Furat and Dirmenci 2009, Bacherpour 2010, Torlak et al. 2010, Bulut and Y1lmaz 2010, Celep and Doğan 2010, İpek and Gürbüz 2010, Y1lmaz et al. 2010, Bacherpour et al. 2011, Çiçek and Ketenoğlu 2011, Güner 2012, Tel 2012, Özçelik 2012, Çiçek and Yaprak 2013, Celep et al. 2015, Dinç and Doğu 2016, Özuslu and Öztekin 2008, Dirmenci et al. 2010, Dirmenci et al. 2011, Öztürk et al. 2011, Güner and Akçiçek 2014, Vural et al. 2015, Akçiçek et al. 2016, Celep 2017, Celep and Dirmenci 2017, Özhatay et al. 2017, Yurteri et al. 2017).

\section{RESULTS AND DISCUSSION}

There are 782 Lamiaceae taxa in Turkey, 346 which are endemic (44.2\% endemic ratio). According to species number, there are 603 Lamiaceae species, 179 subspecies and varietes in Turkey. The largest genera in the country based on the taxon number are Stachys L.(118 taxa), Salvia L.(107 taxa), Sideritis L.(54 taxa), Phlomis L.(53 taxa), Teucrium L. (49 taxa), Thymus L.(47 taxa). The largest 15 genera based on taxon number in Turkey are given in Table 1 (Celep and Dirmenci 2017).

Table 1. The largest 15 genera based on taxon number in Turkish Lamiaceae

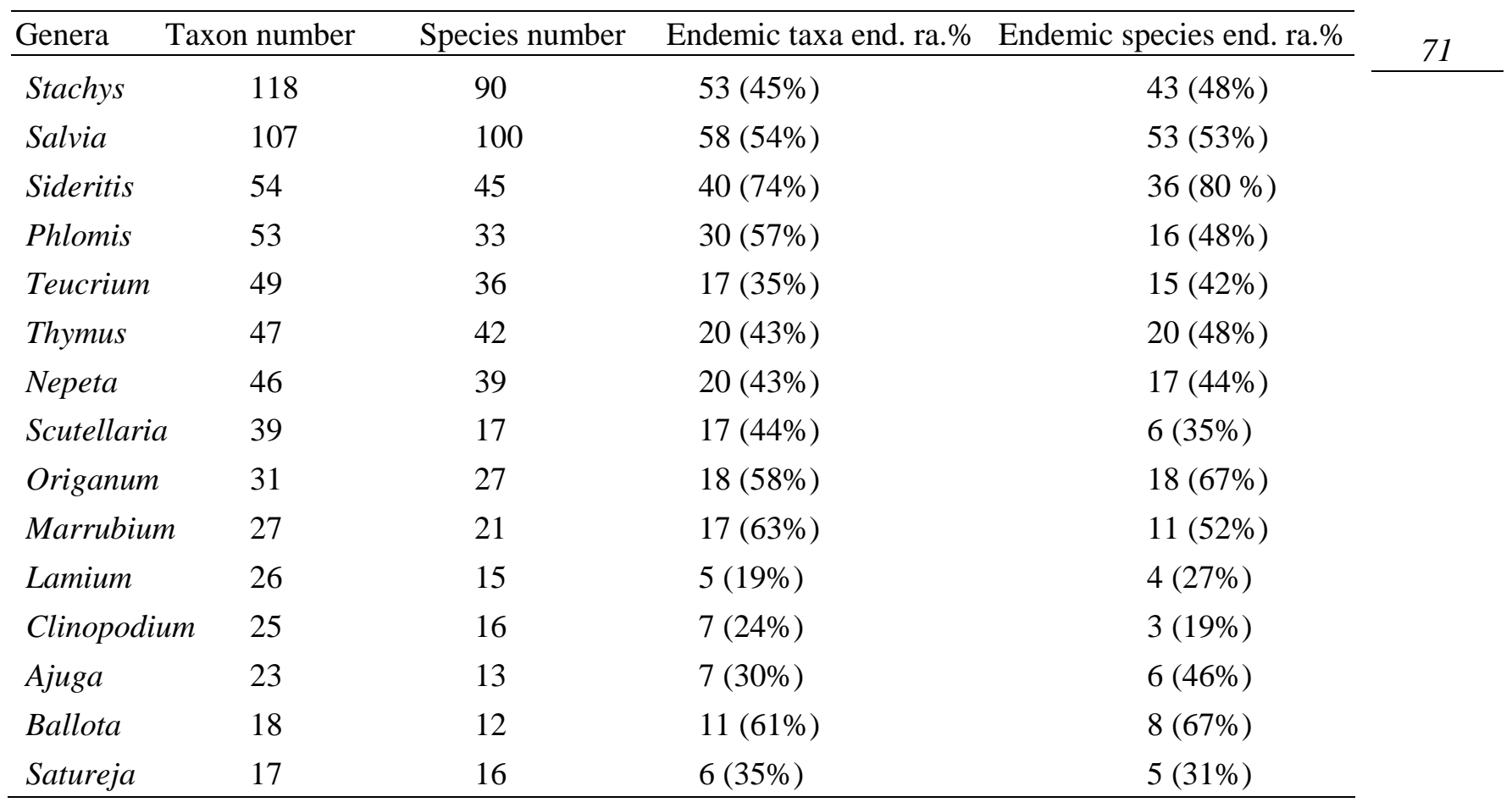

Sideritis Drymosiphon, Marrubium, Ballota, Origanum, Phlomis, Salvia have a large number endemic taxa and species. Their endemism ratio is over $54 \%$ in Turkey. There are two monotypic genera in Turkey as Dorystaechas (endemic) ve Pentapleura (non-endemic). Lophantus has also only one species in Turkey, it is not monotypic genus. Fifteen genera with the highest endemism ratio are given in Table 2 (Celep and Dirmenci 2017). 
Table 2. Fifteen genera with the highest endemism ratio in Lamiaceae in Turkey

\begin{tabular}{llcc}
\hline Genera & $\begin{array}{c}\text { Endemism ratio based } \\
\text { on taxa number\% }\end{array}$ & $\begin{array}{c}\text { Endemism ratio based on } \\
\text { species number \% }\end{array}$ \\
\hline 1 & 100 & 100 \\
2 & Dorystaechas & 100 & 100 \\
3 & Lophantus & 74 & 80 \\
4 & Sideritis & 67 & 75 \\
5 & Drymosiphon & 63 & 52 \\
6 & Marrubium & 61 & 67 \\
7 & Ballota & 58 & 67 \\
8 & Origanum & 57 & 48 \\
9 & Phlomis & 54 & 53 \\
10 & Salvia & 45 & 48 \\
11 & Stachys & 44 & 35 \\
12 & Scutellaria & 43 & 48 \\
13 & Thymus & 43 & 44 \\
14 & Nepeta & 35 & 42 \\
15 & Teucrium & 35 & 31 \\
\hline
\end{tabular}

Result of endangered family of Lamiaceae according to IUCN threat categories are 20 genera in Turkey. These genera; Salvia L.(43 taxa), Stachys L.( 19 taxa), Thymus L. (16 taxa), Sideritis L.(14 taxa), Teucrium L. (10 taxa), Lamium L.(8 taxa), Phlomis L. ve Scutellaria L. ( 7 taxa), Micromeria Bentham ve Nepeta L. ( 6 taxa), Ajuga L. ve Origanum L. ( 5 taxa), Marrubium L. (4 taxa), Acinos Miller, Satureja L. ve Calamintha Miller (2 taxa), Ballota L., Dorystaechas Boiss. \& Heldr. ex Bentham, Cyclotrichium (Boiss.)Manden. \& Scheng. ve Lopanthus Adans.( 1 taxon) (Table 3). 
Table 3. Endangered Lamiaceae genera according to IUCN threat categories in Turkey

\begin{tabular}{|c|c|c|c|}
\hline Genera & $\mathbf{R}$ (critically endangered) & EN (endangered) & VU (vulnerable) \\
\hline Salvia (43 taxa) & 13 & 15 & 15 \\
\hline Stachys (19 taxa) & 3 & 10 & 6 \\
\hline Thymus (16 taxa) & 7 & 4 & 5 \\
\hline Sideritis(14 taxa) & 2 & 3 & 9 \\
\hline Teucrium(10 taxa) & 4 & 3 & 3 \\
\hline Lamium (8 taxa) & 2 & 3 & 3 \\
\hline Phlomis (7 taxa) & 1 & 2 & 4 \\
\hline Scutellaria (7 taxa) & 1 & 3 & 3 \\
\hline Micromeria (6 taxa) & 2 & 2 & 2 \\
\hline Nepeta (6 taxa) & 3 & 2 & 1 \\
\hline Ajuga (5 taxa) & - & 4 & 1 \\
\hline Origanum (5 taxa) & 1 & 3 & 2 \\
\hline Marrubium (4 taxa) & 1 & 2 & 1 \\
\hline $\operatorname{Acinos}(2$ taxa) & - & 2 & - \\
\hline Satureja (2 taxa) & 2 & - & - \\
\hline Calamintha (2 taxa) & - & 1 & 1 \\
\hline Ballota (1 taxa) & - & - & 1 \\
\hline Dorystaechas (1 taxa) & - & - & 1 \\
\hline Cylotrichium (1 taxa) & - & - & 1 \\
\hline Lopanthus (1 taxa) & 1 & - & - \\
\hline
\end{tabular}

Endangered and vulnerable family of Lamiaceae according to IUCN threat categories are 160 taxa in country (Appendix, Table 1).

CR (critically endangered) 43 ( 42 endemic / 1 non-endemic)

EN (endangered) 59 (57 endemic / 2 non-endemic)

VU (vulnerable) 58 (52 endemic / 6 non-endemic)

Threat categories of the largest five genera in the country based on the taxon number are;

Salvia (43 taxa) / $13 \mathrm{CR}$ (critically endangered) / $15 \mathrm{EN}$ (endangered)) / $15 \mathrm{VU}$ (vulnerable)

Stachys (19 taxa) / 3 CR (critically endangered) / 10 EN (endangered)) / 6 VU (vulnerable)

Thymus (16 taxa) / 7 CR (critically endangered) / 4 EN (endangered)) / 5 VU (vulnerable)

Sideritis (14 taxa) / 2 CR (critically endangered) / 3 EN (endangered)) / 9 VU (vulnerable)

Teucrium (10 taxa) / 4 CR (critically endangered)/ 3 EN (endangered)) / 3 VU (vulnerable) 
Figure 2. A. Salvia aytachii Vural\&Adigüzel

B. Salvia kronenburgii Rech. fil. (Nasip Demirkuş)

C. Salvia fruticosa Mill. (Ester Inbar)

D. Stachys distans Bentham var. cilicica Bhattacharjee \& Hub.-Mor.

E. Stachys bayburtensis Bhattacharjee \& Hub.-Mor.

F. Thymus cariensis Hub.-Mor. \& Jalas
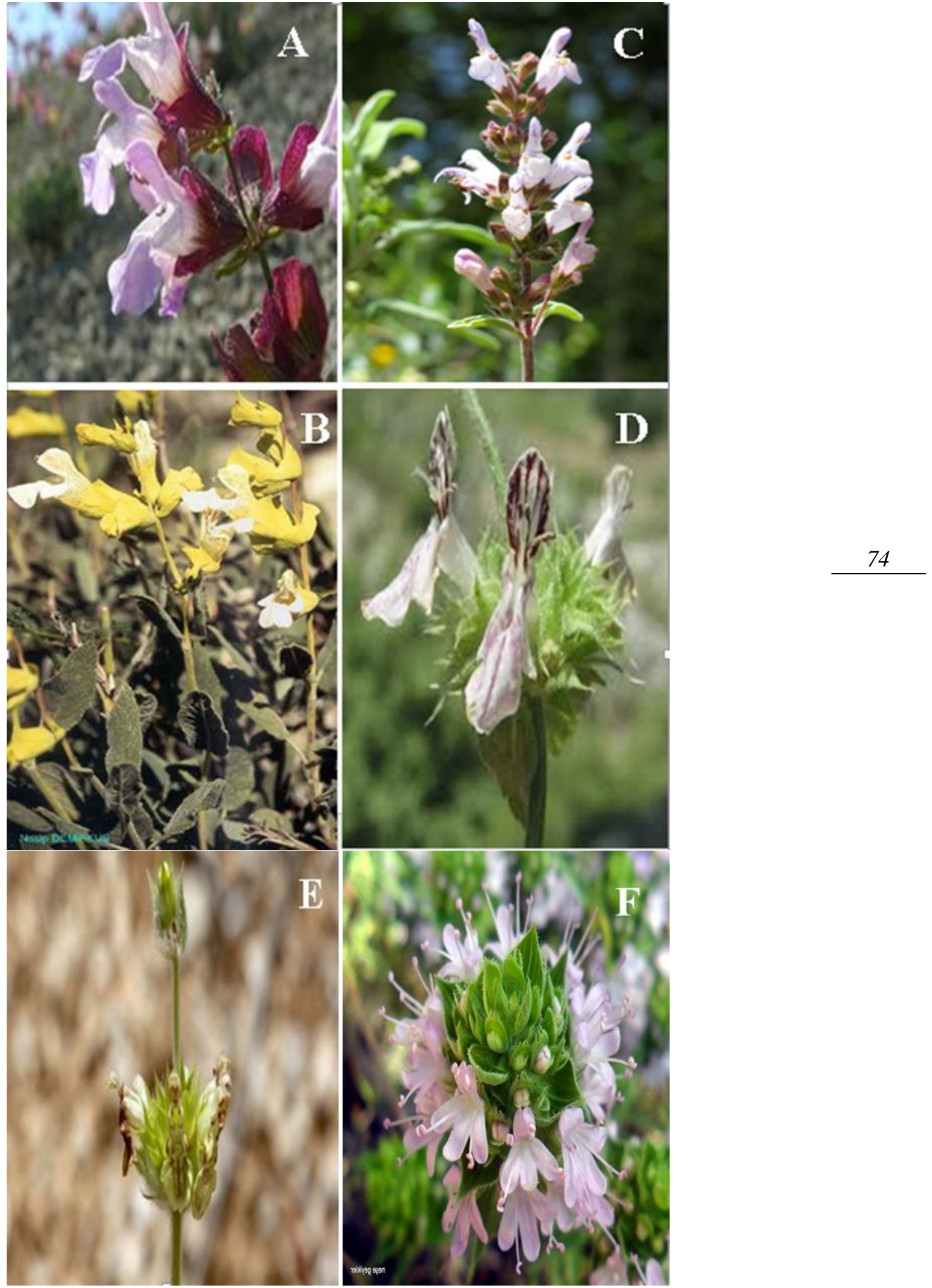
Figure 3. A. Sideritis akmanii Z. Aytaç, M. Ekici \& A. Dönmez

B. Phlomis amanica Vierh.

C. Origanum solymicum P.H. Davis

D. Lamium microphyllum Boiss.

E. Teucrium cavernarum P.H. Davis

F. Thymus pulvinatus Celak.
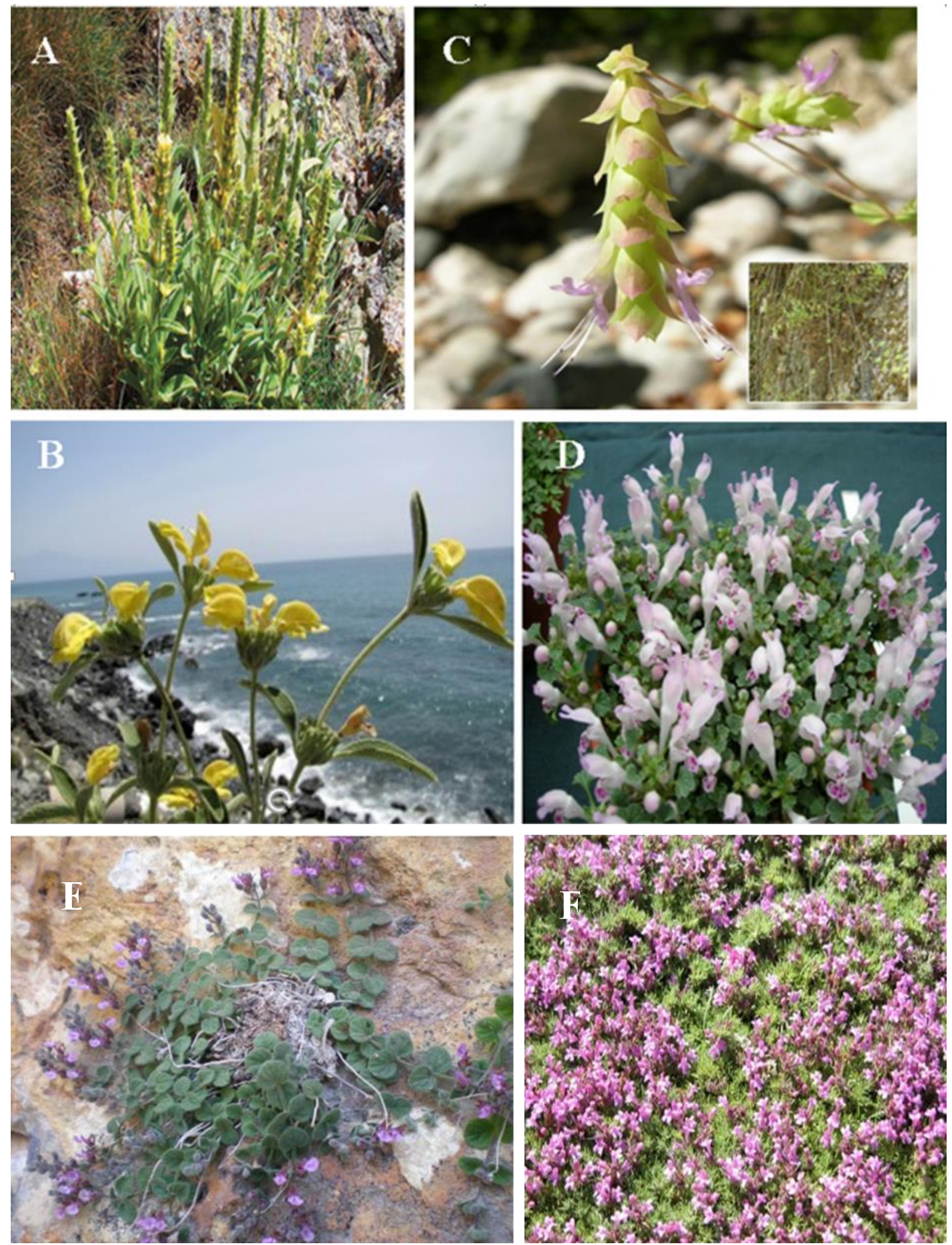
Phytogeographic distribution of Turkish Lamiaceae taxa are 293 taxa in the Mediterranean (\% 37.4), 267 taxa in the Irano-Turanian (\%36.7), 90 taxa in the Euro-Siberian (\% 11.5) phytogeographic region, and 112 taxa in Unknown or Multiregional (\%14.3) phytogeographical elements (Celep and Dirmenci 2017) .

Endangered endemic and rare taxa of Lamiaceae genera are phytogeographic region in Turkey (Table 4).

Mediterranean elements 79 taxa, 74 endemic / 5 non-endemic

İrano-Turanian elements 63 taxa, 58 endemic /5 non-endemic

Euro-Siberian elements 6 taxa, 5 endemic / 1 non-endemic

Unknown or Multiregional elements 11 taxa, 11 endemic

Table 4. Phytogeographic distribution of endangered Lamiaceae genera based on taxon number and Endemism status

\begin{tabular}{|c|c|c|c|c|c|}
\hline Genera (Taxon number) & Irano-Turanian el. & Mediterranean el & I. Euro-Siberian el. & Unknown or Multiregional & Endemic \\
\hline Salvia (43) & 18 & 19 & 1 & 5 & 37 \\
\hline Stachys (19) & 10 & 7 & - & 2 & 18 \\
\hline Thymus (16) & 6 & 6 & - & 4 & 14 \\
\hline Sideritis (14) & 2 & 12 & - & - & 14 \\
\hline Teucrium(10) & 3 & 7 & - & - & 9 \\
\hline Lamium (8) & 1 & 4 & 3 & - & 7 \\
\hline Phlomis (7) & 4 & 3 & - & - & 7 \\
\hline Scutellaria (7) & 4 & 3 & - & - & 7 \\
\hline Micromeria (6) & 1 & 4 & 1 & - & 6 \\
\hline Nepeta (6) & 2 & 4 & - & - & 6 \\
\hline Ajuga (5) & 3 & 2 & - & - & 5 \\
\hline Origanum (5) & 1 & 4 & - & - & 5 \\
\hline Marrubium (4) & 2 & 1 & 1 & - & 4 \\
\hline Acinos (2) & - & 2 & - & - & 2 \\
\hline Satureja (2) & 1 & 1 & - & - & 2 \\
\hline Calamintha (2) & 1 & 1 & - & - & 2 \\
\hline Ballota (1) & 1 & - & - & - & 1 \\
\hline Dorystaechas(1) & - & 1 & - & - & 1 \\
\hline Cylotrichium (1) & 1 & - & - & - & 1 \\
\hline Lopanthus (1) & 1 & - & - & - & 1 \\
\hline
\end{tabular}

In conclusion, according to IUCN threat categories, 160 endemic and rare taxa of Lamiaceae are endangered in Turkey. 43 taxa (26.87\%) are critically endangered (CR) and 59 taxa $(36.87 \%)$ endangered (EN). Total 102 taxa (63.75\%) are endangered and critically 
endangered. 58 taxa (36.25\%) are vulnerable. Phytogeographic distribution of endemic and rare Turkish Lamiaceae taxa are 79 taxa (49.37 \%), in the Mediterranean, 63 taxa (39.37\%) in the Irano-Turanien, 5 taxa $(3.12 \%)$ in the Euro-Siberian phytogeographic region, and 11 taxa $(6.8 \%)$ in unknown or multiregional phytogeographical elements. 


\section{REFERENCES}

AKÇIÇEK E., VURAL M., 2007, Kumular Dağı (Afyonkarahisar)' nın Endemik ve Nadir Bitkileri, Balıkesir Üniversitesi Fen Bilimleri Dergisi, 9 (2), 78-86.

AKÇİÇEK E., FIRAT M., GÜNER Ö., 2016, Stachys hakkariensis (Lamiaceae), A New Species from Eastern Anatolia (Turkey) Belonging to Stachys sect. Olisia, Phytotaxa, 257 (2), 167-173.

ALAN S., OCAK A., 2009, Taxonomical and Morphological Studies on The Genus Calamintha Miller (Lamiaceae) in Turkey, Biodicon, 2 (2), 125-143.

ANONIM 1996, Türkiye Bitki Genetik Çeşitliliğinin Yerinde (Insitu) Korunması Ulusal Planı, Çevre Bakanlığı, Tarım ve Köy İşleri Bakanlığı, Orman Bakanlığı, Ankara.

AYTAÇ Z., AKSOY A., 2000, A New Sideritis Species (Labiatae) from Turkey, Flora Mediterranea, 10, 181-184.

BACHERPOUR S., 2010, Taxonomic Studies on The Genus Salvia L. (Labiatae) in Central Anatolia, Turkey, Doktora Tezi, Middle East Techinacal University The Graduate School of Natural and Applied Sciencis.

BACHERPOUR S., CELEP F., KAHRAMAN A., DOĞAN M., 2011, Salvia brachyantha subs. tankutiana (Lamiaceae), A New Subspecies from Central Anatolia, Turkish Journal of Botany, 35, 343-350.

BULUT Z., YILMAZ H., 2010, The Current Situation of Threatened Endemic Flora in Turkey: Kemaliye (Erzincan) Case, Pak. Journal of Botany, 42 (2); 711-719.

CELEP F., DOĞAN M., 2010, Salvia ekimiana (Lamiaceae), A New Species from Turkey, Ann. Bot. Fennici, 47, 63-66.

CELEP F., DİRMENCİ T., GÜNER Ö., 2015, Salvia hasankeyfence (Lamiaceae), A New Species from Hasankeyf (Batman, South-eastern Turkey), Phtytotaxa, 227 (3), 289-294.

CELEP F., 2017, Lamium bilgilii (Lamiaceae), A New Species from South-western Turkey (Burdur-Muğla), Phytotaxa, 312 (2), 263-270.

CELEP F., DİRMENCİ T., 2017, Systematic and Biogeographic Overview of Lamiaceae in Turkey, Naturel Volatiles \& Essential Oils, 4 (4), 14-27.

Çİ̧EK M., KETENOĞLU O., 2011, Scutellaria anatolica A New Species from Turkey, Ann. Bot. Fennici, 48, 276-279.

ÇİÇEK M., YAPRAK A. E., 2013, Scutellaria yildirimlii (Lamiaceae), A New Species from Turkey, Phytotaxa, 138 (1), 53-58.

DAVIS P.H., 1982, Flora of Turkey and The East Aegean Islands, Vol. 7., Edinburg at the University Press, UK., 0- 85224- 396 -0.

DAVIS P.H., MILL R.R., TAN K., 1988, Flora of Turkey and The East Aegean Islands, Vol. 10.,( Supplement 1), Edinburg at the University Press, UK., 0- 85224- 559- 9.

DİNÇ M., DOĞU S., 2016, Teucrium pruniosum var. aksarayense var. nov. (Lamiacaea) from Central Anatolia, Modern Phytomorphology, 9, 13-17.

DİRMENCİ T., 2003, Türkiye'de Yetişen Nepeta L. (Lamiaceae) Türleri Üzerinde Taksonomik Araştırmalar, Doktora Tezi, Balıkesir Üniversitesi Fen Bilimleri Enstitüsü Biyoloji Eğitimi Anabilim Dalı. 
DİRMENCİ T., YILDIZ B., HEDGE I. C., FIRAT M., 2010, Lophanthus (Lamiaceae) in Turkey: A new Generic Record and A new Species, Turkish Journal Of Botany, 34, 123 129.

DİRMENCİ T., YILDIZ B., AKÇIÇCEK E., MARTIN E., DÜNDAR E., 2011, Stachys vuralii (Lamiaceae), A New Species from North Anatolia, Turkey, Ann. Bot. Fennici, 48, 401408.

DÖNMEZ A.A., 2001, A New Turkish Species of Salvia L. (Lamiaceae), Botanical Journal of Linnean Society, 137, 413-416.

EKİM T., GÜNER A., 2000, The Floristic Richness of Turkey, Bentham-Moxon Trust, Blackwell Publishers, Oxford, UK., Malden, USA.

EKİM T., KOYUNCU M.,VURAL M., DUMAN H., AYTAÇ Z., ADIGÜZEL N., 2000, Türkiye Bitkileri Kırmızı Kitabı, Türkiye Tabiatını Koruma Derneği, Van Yüzüncü Yıl Üniversitesi, Barışcan Ofset, Ankara, Türkiye, 975- 93611- 0- 8.

EKİM T., 2009, Türkiye'nin Nadir Endemikleri, Türkiye İş Bankası Kültür Yayınları, Sertifika no 11213, Özgün Ofset, İstanbul, Türkiye, 978-9944-88-648-2.

FIRAT M., DİRMENCİ T., 2009, Clinopodium hakkaricum (Lamiaceae), A New Species from Turkey, Ann. Bot. Fennici, 46, 451-455.

GÜNER A., ÖZHATAY N., EKİM T., BAŞER K.H.C., 2000, Flora of Turkey and The East Aegean Islands, Vol. 11. ( Supplement 2), Edinburg at the University Press,UK.,0- 0 7486- 1409- 5.

GÜNER A., 2012, Türkiye Bitkileri Listesi (Damarlı Bitkiler), Flora Araştırmaları Derneği, Nezahat Gökyiğit Botanik Bahçesi Yayınları Flora Dizisi 1, Namaş Matbaacılık, İstanbul, Türkiye, 978-605-60425-7-7.

GÜNER Ö., AKÇIÇEK E., 2014, Ulus Dağı' nın (Balıkesir-Türkiye) Endemik ve Nadir Bitkileri, Bağ Bahçe Bilim Dergisi, 1 (3), 32-38.

İPEK A., GÜRBÜZ B., 2010, Türkiye Florasında Bulunan Salvia Türleri ve Tehlike Durumları, Tarla Bitkileri Merkez Araştırma Enstitüsü Dergisi, 19 (1-2), 30-35.

ÖZÇELIKK H.,2012, The Endemic Plant Taxa of The Köprülü Kanyon National Park and Its Surroundings (Antalya-Isparta), 16 (3), 279-296.

ÖZHATAY N., KÜLTÜR Ş., GÜRDAL B., 2017, Check- list of Additional Taxa to the Supplement Flora of Turkey VIII, 2017, İstanbul Journal of Pharmacy, 47 (1), 30-44.

ÖZTÜRK M., UYSAL İ., KARABACAK E., ÇELİK S., 2011, Plant Species Microendemism, Rarity and Conservation ofPseudo-alpine Zone of Kazdağı (Mt. Ida) National ParkTurkey, Procedia Social and Behavioral Science, 19, 778-786.

ÖZUSLU E., ÖZTEKİN M., 2008, A New Localization for Teucrium paederotoides Boiss. et Hausskn. (Lamiaceae), Biodicon, 1(2), 86-90.

TARIMCILAR G., 1998, Karadeniz’ de Yayılışı olan Mentha L. Türleri Üzerinde Korolojik, Morfolojik, Anatomik, Sitolojik, Ekolojik ve Kimyasal Araştırmalar, Doktora Tezi, Uludağ Üniversitesi Fen Bilimleri Enstitüsü, Biyoloji Anabilim Dalı, Bursa.

TEL A. Z., 2012, Bazı Endemik Bitkilerin Kütahya'daki (Türkiye) Yayılış Alanları ve Yeni IUCN Tehlike Kategorilerine Göre Yeniden Değerlendirilmesi, Artvin Çoruh Üniversitesi Orman Fakültesi Dergisi, 13 (1), 88-108 
TORLAK H., VURAL M., AYTAÇ Z., 2010, Endemic Plants of Turkey, Ministry of Culture and Tourism, Pelin Ofset, Ankara, Türkiye, 978-975-17-3595-9.

VURAL M., DUMAN H., DİRMENCİ T., ÖZCAN T., 2015, A New Species of Teucrium sect. Stachyobotrys (Lamiaceae) from the South of Turkey, Turkish Journal of Botany, 39, 318-324.

YILMAZ Ö., DAŞKIN R., KAYNAK G., 2010, Stachys pseudobombycina sp. nov. (Lamiaceae) from South Anatolia, Turkey, Nordic Journal of Botany, 28, 341-343.

YURTERİ E., ÖZCAN A., SEYIS F., KEVSEROĞLU K., 2017, Characterization of some Lamiaceae Species Distributed in The Rize Province, Turkey, International Journal of Plant Breeding and Crop Science, 4 (3), 300-307. 


\section{Appendix I.}

Table 1. Endangered Lamiaceae taxa according to IUCN threat categories in Turkey and Endemism status

\begin{tabular}{|c|c|c|c|}
\hline Taxa & Endemism & Threat categories & \\
\hline Acinos troodi (Post) Leblebici subsp. grandiflorus Hartvig \& Strid & $\mathrm{E}$ & EN & \\
\hline Acinos troodi (Post) Leblebici subsp. vardaranus Leblebici & $\mathrm{E}$ & EN & \\
\hline Ajuga davisiana Kit Tan \& Y1ldız & $\mathrm{E}$ & EN & \\
\hline Ajuga postii Briq. & $\mathrm{E}$ & VU & \\
\hline Ajuga relicta $\mathrm{P} . \mathrm{H}$. Davis & $\mathrm{E}$ & EN & \\
\hline Ajuga vestita Boiss. & $\mathrm{E}$ & EN & \\
\hline Ajuga xylorrhiza Kit Tan & $\mathrm{E}$ & EN & \\
\hline Ballota macrodonta Boiss. \& Bal. & $\mathrm{E}$ & VU & \\
\hline Calamintha caroli-benricana Kit Tan \& Sorger & $\mathrm{E}$ & EN & \\
\hline Calamintha tauricola P.H. Davis & $\mathrm{E}$ & VU & \\
\hline Cyclotrichium niveum (Boiss.) Manden \& Scheng. & $\mathrm{E}$ & VU & \\
\hline Dorystaechas hastataBoiss. \& Heldr. ex Bentham & $\mathrm{E}$ & VU & \\
\hline Lamium armenum Boiss. subsp. sintenisii R. Mill & $\mathrm{E}$ & VU & \\
\hline Lamium bilgilii Celep & $\mathrm{E}$ & $\mathrm{CR}$ & \\
\hline Lamium demirizii A. Khohhr. & $\mathrm{E}$ & EN & \\
\hline Lamium microphyllum Boiss. & $\mathrm{E}$ & VU & \\
\hline Lamium purpureum L. var. aznavourii Gand. Ex Aznav. & $\mathrm{E}$ & $\mathrm{CR}$ & 81 \\
\hline Lamium sandrasicum P.H. Davis & $\mathrm{E}$ & EN & \\
\hline Lamium verronicifolium Bentham & $\mathrm{E}$ & VU & \\
\hline Lamium violaceo-velutinum A. Khohhr. & - & EN & \\
\hline Lopanthus turcicus Dirmenci, Yıldız \& Hedge (B9) & $\mathrm{E}$ & $\mathrm{CR}$ & \\
\hline Marrubium bourgaei Boiss. subsp. caricum P.H. Davis & $\mathrm{E}$ & $\mathrm{CR}$ & \\
\hline Marrubium cephalanthus Boiss. \& Noe subsp. montanum Akgül\&Ketenoğlu & $\mathrm{E}$ & EN & \\
\hline Marrubium vanense Hub.-Mor. & $\mathrm{E}$ & EN & \\
\hline Marrubium vulcanicum Hub.-Mor. & $\mathrm{E}$ & VU & \\
\hline Micromeria carica P.H. Davis & $\mathrm{E}$ & EN & \\
\hline Micromeria cilicica Hausskn. ex P.H. Davis & $\mathrm{E}$ & EN & \\
\hline Micromeria cristata (Hampe) Griseb. subsp. carminea (P.H. Davis) P.H. Davis & $\mathrm{E}$ & VU & \\
\hline Micromeria cymuligera Boiss. \& Hausskn. & $\mathrm{E}$ & VU & \\
\hline Micromeria dolichodonta P.H. Davis & $\mathrm{E}$ & $\mathrm{CR}$ & \\
\hline Micromeria fruticosa (L.) Druce subsp. giresunica P.H. Davis & $\mathrm{E}$ & $\mathrm{CR}$ & \\
\hline Nepeta baytopii Hedge \& Lamond & $\mathrm{E}$ & EN & \\
\hline Nepeta conferta Hedge \& Lamond & $\mathrm{E}$ & $\mathrm{CR}$ & \\
\hline Nepeta crinita Montbret \& Aucher ex Bentham & $\mathrm{E}$ & EN & \\
\hline Nepeta nuda L. subsp. glandulifera Hub.-Mor. \& Davis & $\mathrm{E}$ & $\mathrm{CR}$ & \\
\hline Nepeta phyllochlamys P.H. Davis & $\mathrm{E}$ & VU & \\
\hline
\end{tabular}


Nepeta tumeriana B. Y1ld1z \& T. Dirmenci

Origanum boissieri Ietswaart

Origanum husnucan-baseri H. Duman, Z. Aytaç \& A. Duran

Origanum micranthum Vogel

Origanum munzurense Kit Tan \& Sorger

EN

Origanum solymicum P.H. Davis

EN

Phlomis amanica Vierh.

EN

Phlomis angustissima Hub.-Mor.

Phlomis brunneogaleata Hub.-Mor.

Phlomis grandiflora H.S. Thompson var. fimbrilligera (Hub.-Mor.) Hub.-Mor. E

Phlomis integrifolia Hub.-Mor.

Phlomis physocalyx Hub.-Mor.

Phlomis sintenisii Rech. fil.

Salvia adenocaulon P.H. Davis

Salvia adenophylla Hedge \& Hub.-Mor.

Salvia albimaculata Hedge \& Hub.-Mor.

EN

Salvia anatolica Hamzaoğlu \& A. Duran

Salvia aramiensis Rech. f.

Salvia aucheri Bentham var. aucheri

Salvia aucheri Bentham var. canescens Boiss. \& Heldr.

Salvia aytachii $\mathrm{M}$. Vural \& N. Adıg̈zel

EN

Salvia ballsiana ( Rech. f.) Hedge

$\mathrm{CR}$

Salvia brachyanth (Bordz.) Pobed subsp. tankutiana Bagherpour,Celep, Kahraman \& Doğa E

EN

Salvia cassia G. Samuelsson ex Rech. f.

Salvia cedronella Boiss.

EN

Salvia cerino-pruinosa Rech. var. elazigensis A. Karaman, F. Celep \& Dogan

EN

Salvia chionantha Boiss

$\mathrm{VU}$

Salvia chrysophylla Stapf

Salvia cilicica Boiss. \& Kotschy

EN

Salvia ekimiana F. Celep \& Doğan

EN

Salvia eriophora Boiss. \& Kotschy ex Boiss.

EN

Salvia freyniana Bornm.

CR

Salvia fruticosa Mill

Salvia halophila Hedge

EN

Salvia hasankeyfense Dirmenci, Celep \& Ö. Güner

CR

Salvia hedgeana Dönmez

$\mathrm{CR}$

Salvia heldreichiana Boiss. ex DC.

$\mathrm{VU}$

Salvia kronenburgii Rech. fil.

EN

Salvia kurdica Boiss. \& Hohen. Ex Benth.

Salvia marashica A. İlçim, F. Celep \& Doğan

CR

Salvia modesta Boiss.

EN

Salvia nutans L. 
Salvia nydeggeri Hub.-Mor.

E

EN

Salvia odontochlamys Hedge

CR

Salvia pilifera Montbr. \& Auch.

$\mathrm{VU}$

Salvia pisidica Boiss. \& Hohen. Ex Benth.

VU

Salvia pomifera $\mathrm{L}$.

Salvia potentillifolia Boiss. \& Hohen. Ex Benth.

$\mathrm{VU}$

Salvia pseudeuphratica Rech.

CR

Salvia quezelii Hedge \& Afzal-Rafii

Salvia sericeo-tomentosa Rech. fil. var. sericeo-tomentosa

CR

Salvia sericeo-tomentosa Rech. fil. var. hatayica F. Celep \& Doğan

CR

Salvia smyrnaea Boiss.

CR

Salvia tigrina Hedge \& Hub.-Mor.

EN

Salvia tobeyi Hedge

CR

Salvia vermifolia Hedge \& Hub.-Mor.

EN

Satureja aintabensis P.H. Davis

Satureja amani P.H. Davis

Scutellaria glaphyrostachys Rech. fil.

Scutellaria orientalis L. subsp. carica Edmondson

Scutellaria orientalis L. subsp. porphyrostegia Edmondson

Scutellaria orientalis L. subsp. sintenisii (Hasskn. Ex Bornm.) Edmondson

Scutellaria orientalis L. subsp. tortumensis Kit Tan \& Sorger

Scutellaria rubicunda Hornem. subsp. pannosula (Rech. fil.) Edmondson

Scutellaria uzundereensis A. Khohhr.

Sideritis akmanii Z. Aytaç, M. Ekici \& A. Dönmez

Sideritis bilgerana P.H. Davis

$\mathrm{CR}$

Sideritis brevibracteata P.H. Davis

Sideritis brevidens P.H. Davis

E

E

E

E

E

E

E

E

E

E

E

E

E

Sideritis cilicica Boiss. \& Bal.

E

E

Sideritis erythrantha Boiss. \& Heldr. apud Bentham var. cedratorum P.H. Davis E

Sideritis gulendamii H. Duman \& F.A. Karavelioğulları E

Sideritis huber-morathii Greuter \& Burdet

Sideritis lycica Boiss. \& Heldr. apud Bentham

Sideritis ozturkii Aytaç \& Aksoy

Sideritis serratifolia Hub.-Mor.

Sideritis trojana Bornm.

Sideritis vulcanica Hub.-Mor.

Sideritis vuralii $\mathrm{H}$. Duman \& Başer

Stachys anamurensis H. Sümbül

Stachys antalyensis Y. Ayaşligil \& P.H. Davis

Stachys bayburtensis Bhattacharjee \& Hub.-Mor. 
Stachys cataonica Bhattacharjee \& Hub.-Mor.

$\mathrm{E}$

Stachys chasmosericea Ayaşlıgil \& P.H. Davis

Stachys choruhensis Kit Tan \& Sorger

Stachys distans Bentham var. cilicica Bhattacharjee \& Hub.-Mor.

Stachys hakkariensis Akçiçek \& Firat

Stachys huber-morathii Bhattacharjee

Stachys inanis Hausskn. \& Bornm.

Stachys munzurdagensis Bhattacharjee

Stachys pseudobombycina Kaynak, Daşkın \& Yılmaz

Stachys pseudopinardii Bhattacharjee \& Hub.-Mor.

Stachys sivasica Kit Tan \& Y1ldı

Stachys subnuda Montbret \& Aucher ex Bentham

Stachys tundjeliensis Kit Tan \& Sorger

Stachys willemsei Kit Tan \& Hedge

Teucrium aladagense Vural \& H. Duman

Teucrium antitauricum T. Ekim

Teucrium cavernarum P.H. Davis

Teucrium ekimii $\mathrm{H}$. Duman

Teucrium leuchophyllum Montbret \& Aucher ex Bentham

Teucrium montbretii Bentham subsp. pamphylicum P.H. Davis

Teucrium odontites Boiss. \& Bal.

Teucrium paederotoides Boiss. \& Hausskn.

Teucrium pruinosum Boiss. var. aksarayense M. Dinç \& S. Doğu

Teucrium sirnakense Özcan \& Dirmenci

Thymus aznavourii Velen.

Thymus bornmuelleri Velen.

Thymus canoviridis Jalas

Thymus cappadocicus Boiss. var. globifer Jalas

Thymus cappadocicus Boiss. var. pruinosus (Boiss.) Boiss.

Thymus cariensis Hub.-Mor. \& Jalas

Thymus cherlioides Vis. var. isauricus Jalas

Thymus cherlioides Vis. var. oxyodon Jalas

Thymus convolutus Klokov

Thymus leucostomus Hausskn. \& Velen. var. argillaceus Jalas

Thymus leucostomus Hausskn. \& Velen. var. gypsaceus Jalas

Thymus pectinatus Fisch. \& Mey. var. pallasicus (Hayek \& Velen.) Jalas

Thymus praecox Opiz subsp. praecox var. laniger (Borbas) Jalas

Thymus pulvinatus Celak.

Thymus revolutus Celak.

Thymus spathulifolius Hausskn. \& Velen.
$\mathrm{E}$

$\mathrm{E}$

E

$\mathrm{E}$

$\mathrm{E}$

E

E

$\mathrm{E}$

$\mathrm{E}$

E

E

E

$\mathrm{E}$

$\mathrm{E}$

$\mathrm{E}$

$\mathrm{E}$

$\mathrm{E}$

$\mathrm{E}$

$\mathrm{E}$

$\mathrm{E}$

$\mathrm{E}$

E

$-$

E

E

E

E

E

E

-

E

E

E

E

E

E

E

E

E
VU

CR

EN

EN

EN

VU

VU

EN

CR

VU

EN

VU

EN

EN

EN

VU

VU

CR

CR

VU

EN

EN

EN

CR

CR

VU

EN

VU

VU

CR

EN

CR

EN

VU

CR

CR

CR

CR

VU

EN 